\title{
VERTICAL RESOLUTION ENHANCEMENT BY APPLYING POLARIMETRIC SPECTRAL ANALYSIS TECHNIQUES TO MULTIBASELINE INSAR DATA
}

\author{
Stefan Sauer, Kostas Papathanassiou
}

German Aerospace Center (DLR), Microwaves and Radar Institute (HR), Germany, stefan.sauer@dlr.de

\begin{abstract}
Polarimetric spectral analysis techniques have been recently introduced for processing multibaseline interferometric synthetic aperture radar data that are affected by the speckle phenomenon. In this paper, their phase estimation accuracy for retrieving the three-dimensional structure of scatterers from measurements corrupted by multiplicative noise is examined. The performance of the polarimetric beamforming, Capon, and MUSIC algorithms is investigated by Monte Carlo simulations and compared with the polarimetric Cramér-Rao bound. It is demonstrated by the CRLB that radar probing systems possessing polarization diversity offer the potential of considerably improving the information extracted from the observation space enlarged by scattering polarimetry. Moreover, it is shown that the polarimetric algorithms lead to noticeably enhanced estimation precision, in particular in terms of refined resolution, under the condition of polarization diversity.
\end{abstract}

Index Terms - Polarimetric multibaseline interferometric CRLB, polarimetic performance analysis, multi-channel synthetic aperture radar signal processing, multiplicative noise, polarimetric array signal processing techniques.

\section{INTRODUCTION}

In the past, spectral analysis techniques to process single polarization multibaseline interferometric SAR data have been investigated by numerical simulations and the Cramér-Rao lower bound (CRLB) [1,2]. Recently, a new way of processing polarimetric multibaseline InSAR observations has been introduced: Beamforming, Capon, and MUSIC have been adapted to the scenario of scattering polarimetry [3, 4] based on ideas in $[5,6]$. The polarimetric algorithms have been examined using real polarimetric multibaseline InSAR data $[7,8,9]$.

In this paper, the performance of these new methods is evaluated by Monte Carlo simulations and compared with the CRLB extended to the polarimetric case. It is shown that polarimetric analysis techniques enhance significantly the vertical resolution by separating closely located scatterers having diverse polarizations.

\section{POLARIMETRIC SIGNAL MODEL}

The polarimetric multibaseline InSAR data vector $\mathbf{y}(l)$ with $p$ sensors is modeled as

$$
\mathbf{y}(l)=\sum_{m=1}^{N_{s}} \sqrt{\tau_{m}} \mathbf{x}_{m}(l) \odot \mathbf{b}\left(\boldsymbol{\theta}_{m}\right)+\mathbf{n}(l)
$$

where $L$ is the number of statistically independent samples, $l=1, \ldots, L$, and $\odot$ the Schur-Hadamard product (elementwise multiplication). The polarimetric measurement vector $\mathbf{y}(l) \in \mathbb{C}^{\tilde{p}}$ with $\tilde{p}$ being the product of the number of probing devices $p$ and the polarization number $N_{p o l}, \tilde{p}=p N_{p o l}$. For classical single polarization observations $N_{p o l}=1$, and $N_{\text {pol }}=3$ for fully polarimetric configurations with equal cross-polarizations. In the following, the case $N_{p o l}=3$ is considered, but the developed concept is also valid for quadpolarized data $\left(N_{p o l}=4\right)$ and in dual-polarized scenarios $\left(N_{p o l}=2\right)$. As an example, the polarimetric data vector in the lexicographic basis has the shape

$$
\begin{aligned}
\mathbf{y}(l)= & {\left[s_{H H, 1}(l), \ldots, s_{H H, p}(l), \sqrt{2} s_{H V, 1}(l), \ldots,\right.} \\
& \left.\sqrt{2} s_{H V, p}(l), s_{V V, 1}(l), \ldots, s_{V V, p}(l)\right]^{T}
\end{aligned}
$$

where $s_{\mu \nu, i}(l), i=1, \ldots, p$, is the data captured by sensor $i$ in $\mu \nu$ polarization, $\mu, \nu \in\{H, V\}$. The polarimetric reflectivity $\tau_{m}$ of the $m$ th source is assumed to be deterministic as well as the number of backscattering sources $N_{s}$. The multibaseline polarimetric interferometric (MBPI) steering vector $\mathbf{b}\left(\boldsymbol{\theta}_{m}\right), \mathbf{b}\left(\boldsymbol{\theta}_{m}\right) \in \mathbb{C}^{\tilde{p}}$ is a function of the vector of parameters $\boldsymbol{\theta}_{m}$, that are modeled as deterministic quantities. The subindex $m$ is dropped in the following for clarity reasons. The MBPI steering vector is a linear combination of three vectors $\mathbf{a}_{\xi}(\varphi) \in \mathbb{C}^{\tilde{p}}, \xi=1, \ldots, N_{p o l}$, each of them related to one particular polarization

$$
\begin{aligned}
\mathbf{b}(\varphi, \mathbf{w}) & =w_{1} \mathbf{a}_{1}(\varphi)+w_{2} \mathbf{a}_{2}(\varphi)+w_{3} \mathbf{a}_{3}(\varphi) \\
& =\left[w_{1} \mathbf{a}^{T}(\varphi), w_{2} \mathbf{a}^{T}(\varphi), w_{3} \mathbf{a}^{T}(\varphi)\right]^{T}
\end{aligned}
$$

where $\mathbf{a}(\varphi) \in \mathbb{C}^{p}$ is the uniform linear array (ULA) steering vector

$$
\mathbf{a}(\varphi)=[1, \exp \{j \varphi /(p-1)\}, \ldots, \exp \{j \varphi\}]^{T} .
$$


The interferometric phase $\varphi$ is related to the spatial frequency $\omega, \omega \in[-\pi, \pi)$, by $[1,10,11]$

$$
\varphi=(p-1) \omega .
$$

The weighting coefficients $w_{\xi} \in \mathbb{C}$ form a vector

$$
\mathbf{w}=\left[w_{1}, w_{2}, w_{3}\right]^{T}
$$

that is a unitary scattering mechanism, $\mathbf{w}^{H} \mathbf{w}=1$. It comprises the polarimetric features of the backscattering source. To each phase center belongs one particular scattering mechanism, i.e., one specific polarization combination. The complex vector $\mathbf{w}$ is uniquely defined apart from an exponential term $\exp (j \psi)$ with $\psi \in \mathbb{R}$, that cancels out when forming $\mathbf{b}\left(\boldsymbol{\theta}_{m}\right) \mathbf{b}^{H}\left(\boldsymbol{\theta}_{m}\right)$. Hence, without loss of generality $\Re\left(w_{1}\right)$ and $\Im\left(w_{1}\right)$ can be considered to be fixed due to the normalization of $\mathbf{w}$, and the exponential term, respectively. In such a way, the polarimetric model is a generalization of the single polarization multibaseline InSAR model introduced in [1,2], and conforms to the conventional single polarization model for $N_{s}=1$.

The MBPI steering vector can be written in matrix notation as

$$
\mathbf{b}(\varphi, \mathbf{w})=\mathbf{B}(\varphi) \mathbf{w}
$$

with the steering vector matrix $\mathbf{B}(\varphi) \in \operatorname{Mat}_{\tilde{p}, N_{p o l}}(\mathbb{C})$

$$
\mathbf{B}(\varphi)=\left[\begin{array}{ccc}
\mathbf{a}(\varphi) & \mathbf{0} & \mathbf{0} \\
\mathbf{0} & \mathbf{a}(\varphi) & \mathbf{0} \\
\mathbf{0} & \mathbf{0} & \mathbf{a}(\varphi)
\end{array}\right]
$$

The multiplicative noise $\mathbf{x}_{m}(l) \in \mathbb{C}^{\tilde{p}}$ associated with the $m$ th source is modeled as a stationary, circular Gaussian random vector with zero mean, unit variance, and covariance matrix $\mathbf{C}_{m}$. The random processes $\mathbf{x}_{m}\left(l_{1}\right)$ and $\mathbf{x}_{m}\left(l_{2}\right)$ are assumed to be independent and identically distributed for different realizations $l_{1} \neq l_{2}$. For the numerical simulations in section 4 further assumptions about the structure of the speckle covariance matrix are introduced. The additive white Gaussian noise $\mathbf{n}(l) \in \mathbb{C}^{\tilde{p}}$ is assumed to have zero mean and power $\sigma_{n}^{2}$, i.e., $\mathbf{n}(l) \sim \mathcal{N}_{\mathbb{C}}^{\tilde{p}}\left(\mathbf{0}, \sigma_{n}^{2} \mathbf{I}\right)$. Thus, the polarimetric MB InSAR data $\mathbf{y}(l)$ is a circular Gaussian random vector with zero mean and covariance matrix $\mathbf{R}=E\left\{\mathbf{y}(l) \mathbf{y}^{H}(l)\right\}$ with

$$
\mathbf{R}=\sum_{m=1}^{N_{s}} \tau_{m} \mathbf{C}_{m} \odot \mathbf{b}\left(\boldsymbol{\theta}_{m}\right) \mathbf{b}^{H}\left(\boldsymbol{\theta}_{m}\right)+\sigma_{n}^{2} \mathbf{I} .
$$

\section{POLARIMETRIC SPECTRAL ANALYSIS TECHNIQUES}

\subsection{Polarimetric Beamforming}

The beamforming method has been proposed for sensors having polarization diversity on receive in [6]. The spectrum of the polarimetric beamforming is [7]

$$
\hat{P}_{B F}^{P}(\omega)=\frac{\lambda_{\max }\left(\mathbf{B}^{H}(\omega) \hat{\mathbf{R}} \mathbf{B}(\omega)\right)}{p^{2}}
$$

where $\lambda_{\max }(\cdot)$ is the maximum eigenvalue operator. This means that for each frequency $\omega$ the maximal eigenvalue and its corresponding eigenvector of the linear system

$$
\mathbf{B}^{H}(\omega) \hat{\mathbf{R}} \mathbf{B}(\omega) \mathbf{w}_{\max }=\lambda_{\max } \mathbf{w}_{\max }
$$

have to be computed. The frequency estimates $\hat{\omega}=\left[\hat{\omega}_{1}, \ldots, \hat{\omega}_{N_{s}}\right]^{T}$ are given by the locations of the $N_{s}$ maxima of the spectrum.

\subsection{Polarimetric Capon Method}

The Capon algorithm has been extended to the particular scenario of polarization diversity in the receive channel in [6]. The spectrum of the polarimetric Capon method is given by

$$
\hat{P}_{C}^{P}(\omega)=\frac{1}{\lambda_{\min }\left(\mathbf{B}^{H}(\omega) \hat{\mathbf{R}}^{-1} \mathbf{B}(\omega)\right)}
$$

where $\lambda_{\min }(\cdot)$ is the minimum eigenvalue operator. For each frequency $\omega$ the minimal eigenvalue and associated eigenvector of the linear system [7]

$$
\mathbf{B}^{H}(\omega) \hat{\mathbf{R}}^{-1} \mathbf{B}(\omega) \mathbf{w}_{\min }=\lambda_{\min } \mathbf{w}_{\text {min }}
$$

have to be calculated. The inverse matrix $\hat{\mathbf{R}}^{-1}$ exists if the noise term has a positive definite covariance matrix and

$$
L \geq p N_{p o l} .
$$

The frequency estimates $\hat{\boldsymbol{\omega}}=\left[\hat{\omega}_{1}, \ldots, \hat{\omega}_{N_{s}}\right]^{T}$ are related to the positions of the $N_{s}$ largest peaks of the spectrum $\hat{P}_{C}^{P}(\omega)$.

\subsection{Polarimetric MUSIC Algorithm}

The MUSIC approach has been adapted to the special configuration of polarization diversity in the receive antennas in $[5,6]$. The eigendecomposition of $\hat{\mathbf{R}}$ provides the eigenvalues $\hat{\lambda}_{1} \geq \cdots \geq \hat{\lambda}_{\tilde{p}}$ and the eigenvectors $\left(\hat{\mathbf{f}}_{1}, \ldots, \hat{\mathbf{f}}_{N_{s}}\right)$ and $\left(\hat{\mathbf{g}}_{1}, \ldots, \hat{\mathbf{g}}_{\tilde{p}-N_{s}}\right)$ associated with the signal and noise subspace, respectively. Using the matrix $\hat{\mathbf{G}}=\left[\hat{\mathbf{g}}_{1}, \ldots, \hat{\mathbf{g}}_{\tilde{p}-N_{s}}\right] \in$ $\operatorname{Mat}_{\tilde{p}, \tilde{p}-N_{s}}(\mathbb{C})$, the pseudo-spectrum of the polarimetric MUSIC method is [7]

$$
\hat{P}_{M U}^{P}(\omega)=\frac{1}{\lambda_{\min }\left(\mathbf{B}^{H}(\omega) \hat{\mathbf{G}} \hat{\mathbf{G}}^{H} \mathbf{B}(\omega)\right)} .
$$

For each frequency $\omega$ the minimum eigenvalue and its eigenvector of the linear system

$$
\mathbf{B}^{H}(\omega) \hat{\mathbf{G}} \hat{\mathbf{G}}^{H} \mathbf{B}(\omega) \mathbf{w}_{\min }=\lambda_{\min } \mathbf{w}_{\min }
$$

are calculated. The frequency estimates $\hat{\boldsymbol{\omega}}=\left[\hat{\omega}_{1}, \ldots, \hat{\omega}_{N_{s}}\right]^{T}$ correspond to the locations of the $N_{s}$ maxima of the pseudospectrum $\hat{P}_{M U}^{P}(\omega)$. 


\section{NUMERICAL ANALYSIS}

For analyzing the performance of the polarimetric methods and comparing their root mean square error with the CRLB, the following assumptions concerning the multiplicative noise $\mathbf{x}_{m}(l)$ are made. The polarimetric speckle covariance matrix $\mathbf{C}_{m}=E\left\{\mathbf{x}_{m}(l) \mathbf{x}_{m}^{H}(l)\right\} \in \mathbf{M a t}_{\tilde{p}}(\mathbb{R})$ of the $m$ th source is a block matrix of the form

$$
\mathbf{C}_{m}=\left[\begin{array}{lll}
\mathbf{C}_{H H H H, m} & \mathbf{C}_{H H V V, m} & \mathbf{C}_{H H H V, m} \\
\mathbf{C}_{H H V V, m}^{H} & \mathbf{C}_{V V V V, m} & \mathbf{C}_{V V H V, m} \\
\mathbf{C}_{H H H V, m}^{H} & \mathbf{C}_{V V H V, m}^{H} & \mathbf{C}_{H V H V, m}
\end{array}\right] .
$$

The autocorrelation function of each block matrix $\mathbf{C}_{\mu \nu, m} \in$ $\operatorname{Mat}_{p}(\mathbb{R})$ is given by

$\left[\mathbf{C}_{\mu \nu, m}\right]_{s, t}=\left\{\begin{array}{cl}\left(1-\frac{|s-t|}{p-1} b_{\mu \nu, m}\right) d_{\mu \nu, m} & |s-t| \leq \frac{p-1}{b_{\mu \nu, m}} \\ 0 & \text { otherwise }\end{array}\right.$

where $\mu, \nu \in\{H H, V V, H V\}$. The normalized baselines [1] of the like-wise polarization channels are denoted by $b_{H H H H, m}, b_{V V V V, m}, b_{H V H V, m} \in \mathbb{R}$, and the normalized baselines of the cross-polarizations by

$b_{H H V V, m}, b_{H H H V, m}, b_{V V H V, m} \in \mathbb{R}$. The coefficients $d_{H H V V, m}, d_{H H H V, m}, d_{V V H V, m} \in \mathbb{R}$ represent the crosspolarization correlations. Each of these parameters attain values between 0 and 1 . It is assumed that the like-wise polarization correlations $d_{H H H H, m}=d_{V V V V, m}=d_{H V H V, m}=1$. It is clear that this model constitutes a generalization of the single polarization model $[1,2]$, since each block diagonal matrix corresponds to one polarization.

The vector of real-valued unknown parameters $\chi \in$ $\mathbb{R}^{15 N_{s}+1}$ of the covariance matrix (8) is

$$
\begin{aligned}
\chi= & {\left[\varphi_{1}, \ldots, \varphi_{N_{s}}, \tau_{1}, \ldots, \tau_{N_{s}}, \Re\left(w_{2,1}\right), \ldots, \Re\left(w_{2, N_{s}}\right),\right.} \\
& \Im\left(w_{2,1}\right), \ldots, \Im\left(w_{2, N_{s}}\right), \Re\left(w_{3,1}\right), \ldots, \Re\left(w_{3, N_{s}}\right), \\
& \Im\left(w_{3,1}\right), \ldots, \Im\left(w_{3, N_{s}}\right), b_{H H H H, 1}, \ldots, b_{H H H H, N_{s}}, \\
& b_{V V V V, 1}, \ldots, b_{V V V V, N_{s}}, b_{H V H V, 1}, \ldots, b_{H V H V, N_{s}}, \\
& b_{H H V V, 1}, \ldots, b_{H H V V, N_{s}}, b_{H H H V, 1}, \ldots, b_{H H H V, N_{s}}, \\
& b_{V V H V, 1}, \ldots, b_{V V H V, N_{s}}, d_{H H V V, 1}, \ldots, d_{H H V V, N_{s}}, \\
& d_{H H H V, 1}, \ldots, d_{H H H V, N_{s}}, d_{V V H V, 1}, \ldots, d_{V V H V, N_{s}}, \\
& \left.\sigma_{n}^{2}\right]^{T} .
\end{aligned}
$$

They are considered to be deterministic. In the simulation it is assumed that two phase centers are present, $N_{s}=2$, with $\varphi_{1}=0^{\circ}$ and $\varphi_{1}=540^{\circ}$. The standard values of the main simulation parameters are summarized in Table 1. The number of sensors is $p=8$ and the number of independent samples $L=82$. The signal-to-noise ratio is defined by $\mathrm{SNR}_{m}=\tau_{m} / \sigma_{n}^{2}$.

The performance of the polarimetric array processing techniques is investigated utilizing the bias, $\operatorname{bias}\left(\hat{\varphi}_{m}\right)=$ $E\left\{\hat{\varphi}_{m}-\varphi_{m}\right\}$, and the root mean square error, $\operatorname{RMSE}\left(\hat{\varphi}_{m}\right)=$
Table 1. Standard values of main simulation parameters.

\begin{tabular}{|c|c|c|}
\hline & $\begin{array}{c}\text { First source } \\
(m=1)\end{array}$ & $\begin{array}{c}\text { Second source } \\
(m=2)\end{array}$ \\
\hline$\varphi_{m}[\mathrm{deg}]$ & 0.0 & 540.0 \\
\hline $\mathrm{SNR}_{m}[\mathrm{~dB}]$ & 12.0 & 12.0 \\
\hline $\mathbf{w}_{m}$ & {$\left[\begin{array}{c}0.7070 \\
-0.0141 j \\
-0.7070\end{array}\right]$} & {$\left[\begin{array}{c}0.7070 \\
0.0071 \\
0.7070\end{array}\right]$} \\
\hline$b_{H H H H, m}$ & 0.2 & 0.2 \\
\hline$b_{V V V V, m}$ & 0.2 & 0.2 \\
\hline$b_{H V H V, m}$ & 0.2 & 0.2 \\
\hline$b_{H H V V, m}$ & 0.2 & 0.2 \\
\hline$b_{H H H V, m}$ & 0.2 & 0.2 \\
\hline$b_{V V H V, m}$ & 0.2 & 0.2 \\
\hline$d_{H H V V, m}$ & 0.9 & 0.9 \\
\hline$d_{H H H V, m}$ & 0.2 & 0.2 \\
\hline$d_{V V H V, m}$ & 0.2 & 0.2 \\
\hline
\end{tabular}

$\sqrt{E\left\{\left(\hat{\varphi}_{m}-\varphi_{m}\right)^{2}\right\}}$. The numerical simulations are performed with $10^{3}$ Monte Carlo runs. The phase estimation accuracy of the polarimetric algorithms is compared with each other and the CRLB. The impact of one parameter on the performance is examined by varying this particular parameter while keeping the others fixed.

The estimation precision is analyzed by altering the distance between the phase centers, $\Delta \varphi=\varphi_{2}-\varphi_{1}$, with $\varphi_{2}$ in the range of $\left[25^{\circ}, 500^{\circ}\right]$. Firstly, two sources having diverse polarization properties are considered (cf. $\mathbf{w}_{1}$ and $\mathbf{w}_{2}$ in Table 1). When the phase centers are well separated, the polarimetric methods are (almost) efficient (see Figure 1), Capon exhibiting a larger RMSE than beamforming and MUSIC. As the phases get closer together, the CRLB increases and the performance of the polarimetric algorithms degrades: Beamforming diverges from the CRLB for $\Delta \varphi<225^{\circ}$, Capon for $\Delta \varphi<125^{\circ}$, and MUSIC for $\Delta \varphi<50^{\circ}$. The techniques are unbiased for large $\Delta \varphi$ (not reported here), and their biases start differing significantly from $0^{\circ}$ at $\Delta \varphi \approx 225^{\circ}$ (beamforming), $\Delta \varphi \approx 75^{\circ}$ (Capon), and $\Delta \varphi \approx 25^{\circ}$ (MUSIC).

Secondly, it is assumed that the polarization features of the phase centers are similar with

$$
\mathbf{w}_{1}=[0.7070,-j 0.0141,-0.7070]^{T}
$$

and

$$
\mathbf{w}_{2}=[0.7070,0.0070,-0.7070]^{T} .
$$

In the scenario of similar polarizations depicted in Figure 2, the CRLB and the RMS errors increase much faster than in the case of diverse polarizations. The CRLB is above $10^{\circ}$ at $\Delta \varphi<160^{\circ}$ if the phases possess similar polarimetric features, whereas it reaches this barrier at $\Delta \varphi<40^{\circ}$ if the sources have different polarimetric characteristics. In the situ- 


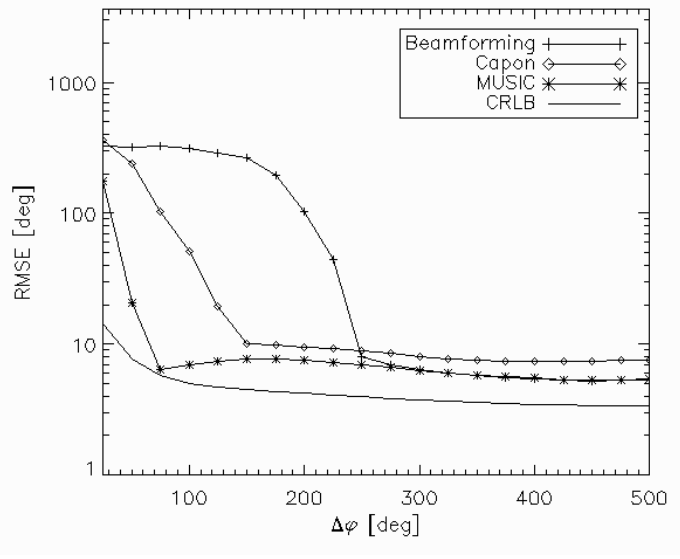

Fig. 1. RMSE of $\hat{\varphi}_{1}$ and $\hat{\varphi}_{2}$ versus $\Delta \varphi$ for diverse polarizations.

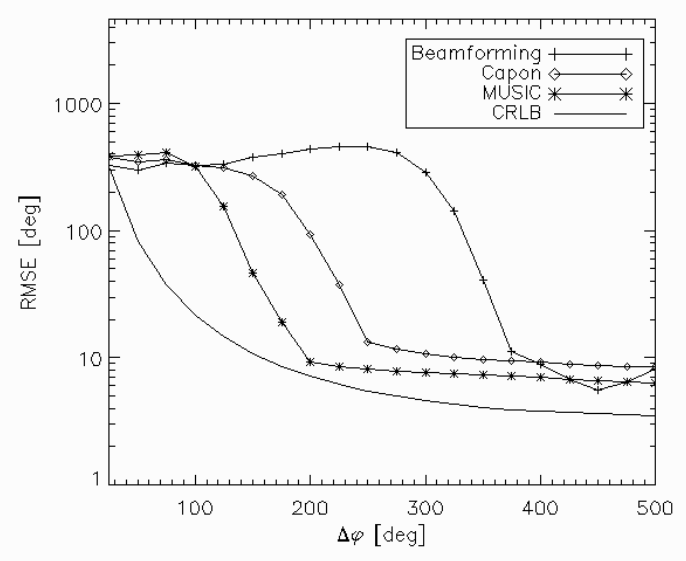

Fig. 2. RMSE of $\hat{\varphi}_{1}$ and $\hat{\varphi}_{2}$ versus $\Delta \varphi$ for similar polarizations.

ation of alike polarizations, the phase estimates of beamforming depart from the CRLB and their RMSEs are more than $10^{\circ}$ for $\Delta \varphi<375^{\circ}$, in the case of Capon at $\Delta \varphi \approx 275^{\circ}$, and for MUSIC at $\Delta \varphi \approx 175^{\circ}$. Similar behavior can be found for the bias not reported here.

Concluding, this analysis demonstrates that under the assumption of phase centers related to diverse polarizations, the performance of polarimetric acquisition systems (as indicated by the CRLB) and the estimation accuracy of polarimetric algorithms are remarkably better than in the case of similar polarizations. Polarization diversity leads to considerably refined resolution.

\section{CONCLUSION}

The performance of polarimetric spectral analysis methods for estimating the vertical structure of scatterers using multi- baseline InSAR data has been investigated. The phase retrieval accuracy has been examined for the polarimetric beamforming, Capon, and MUSIC algorithms in terms of bias and RMSE and compared with the polarimetric CRLB with respect to phase center distance and polarization diversity.

Most interestingly, by reducing the phase center difference it has been shown that the estimation precision can be enhanced considerably under the condition of diverse polarizations compared with the case of similar polarizations. That means that polarimetric systems and processing algorithms have a significantly improved resolution capability over single polarization scenarios.

These findings confirm results obtained by applying the polarimetric spectral analysis techniques to multibaseline InSAR data acquired by the E-SAR system of DLR over forested terrain [12] and urban areas [13].

\section{REFERENCES}

[1] F. Gini, F. Lombardini, and M. Montanari, "Layover solution in multibaseline sar interferometry," IEEE Trans. Aerospace Electronic Systems, vol. 38, no. 4, pp. 1344-1356, Oct. 2002.

[2] F. Gini and F. Lombardini, "Multibaseline cross-track sar interferometry: A signal processing perspective," IEEE AE Systems Magazine, vol. 20, pp. 71-93, August 2005.

[3] S. Sauer, L. Ferro-Famil, A. Reigber, and E. Pottier, "Multibaseline pol-insar analysis of urban scenes for $3 \mathrm{~d}$ modeling and physical feature retrieval at 1-band," in Proceedings of IGARSS 2007, July 2007.

[4] S. Sauer, L. Ferro-Famil, A. Reigber, and E. Pottier, "3d urban remote sensing using dual-baseline pol-insar images at l-band," in Proceedings of IGARSS 2008, July 2008.

[5] R. O. Schmidt, "Multiple emitter location and signal parameter estimation," in Proc. RADC Estimation Workshop, Oct. 1979.

[6] E. R. Ferrara and T. M. Parks, "Direction finding with an array of antennas having diverse polarizations," IEEE Trans. on Antennas and Propagation, vol. 31, pp. 231-236, March 1983.

[7] S. Sauer, Interferometric SAR Remote Sensing of Urban Areas at LBand using Multibaseline and Polarimetric Spectral Analysis Techniques, Ph.D. thesis, 2008.

[8] S. Sauer, L. Ferro-Famil, A. Reigber, and E. Pottier, "3d urban remote sensing using spectral analysis techniques applied to 1-band dualbaseline pol-insar images," in Proc. EUSAR, June 2008.

[9] S. Sauer, L. Ferro-Famil, A. Reigber, and E. Pottier, "Polarimetric dualbaseline insar building height estimation at 1-band," IEEE Geoscience and Remote Sensing Letters, vol. 6, no. 3, pp. 408-412, July 2009.

[10] E. Rodriguez and J. M. Martin, "Theory and design of interferometric synthetic aperture radars," in Proc. Inst. Elect. Eng. F, April 1992.

[11] P. Stoica and R. Moses, Introduction to Spectral Analysis, Prentice Hall, 1997.

[12] S. Sauer, F. Kugler, S.-K. Lee, and K. Papathanassiou, "Polarimetric decomposition applied to $3 \mathrm{~d}$ sar images of forested terrain," in Proc. EUSAR, June 2010.

[13] S. Sauer, L. Ferro-Famil, A. Reigber, and E. Pottier, "Threedimensional imaging and scattering mechanism estimation over urban scenes using dual-baseline polarimetric insar observations at l-band," IEEE Trans. Geoscience Remote Sensing, vol. 49, no. 11, pp. 4616 4629, Nov. 2011. 